\title{
A Pilot Study of the Impact of Repeated Sessions of Virtual Reality on Chronic Neuropathic Pain
}

Ted Jones 1, Rebecca Skadberg 2, Todd Moore 2

1 Pain Consultants of East Tennessee

2 University of Tennessee Knoxville

Abstract - OBJECTIVE: The use of virtual reality (VR) for pain has numerous studies showing effectiveness. However, there has been limited study of its use for chronic pain. METHODOLGY: This pilot study $(\mathrm{N}=10)$ investigated the impact of repeated sessions of a VR application for chronic pain on ten subjects. Impact on pain as well as on psychological variables such as depression, anxiety, catastrophizing, and sense of control over pain was assessed. Subjects underwent three twenty minute sessions of the VR application Cool! on a weekly basis using an Oculus Rift or Vive. The impact of the sessions on pain was assessed at four intervals and psychological data captured at two intervals. RESULTS: Results indicate that the VR sessions provided significant pain relief in all treatment sessions with an average of a $66 \%$ reduction in pain during the VR session and a $45 \%$ reduction in pain immediately after the session. A decrease in pain was reported to last an average of 30 hours after the session. There appeared to be limited if any impact of the VR intervention on chronic pain levels across time. There was no significant impact found for the VR intervention on depression, anxiety, catastrophizing and sense of control over pain. CONCLUSION: Implications for the use of VR on chronic pain conditions are discussed. More frequent VR interventions for chronic pain may be needed to impact pain across time. In addition, VR applications might not be used as an interventional-type in-office treatment as done here but perhaps need to have a skill teaching component or be an application available for in-home and more frequent use.

Index Terms - chronic pain, virtual reality, neuropathic pain, depression, catastrophizing

\section{INTRODUCTION}

Many Americans suffer from chronic pain and much of that pain is under-treated or treated ineffectively [1-3]. Meanwhile, misuse and diversion of opioids used to treat chronic pain conditions have increased 
dramatically in recent years [4]. This leaves those who treat chronic pain conditions with the twin dilemma of working to treat chronic pain conditions in an effective manner while prescribing opioid medications in a very cautious manner, if prescribed at all. Safe, effective alternatives to opioid medications are vitally important to consider and test to assist with the great number of persons with chronic pain conditions who need to be helped without the concerns related to the use of opioid medications.

\subsection{Past Studies}

Virtual reality (VR) applications have been shown to provide significant pain relief for acute pain conditions such as burn pain and wound care [5-15]. These numerous studies have demonstrated that VR is an effective tool in significantly reducing pain in acute pain situations. The "gate theory" of attention is the most widely accepted model in explaining the impact of VR on pain $[16,17]$. Gate theory of attention postulates that VR reduces the perception of pain by absorbing and diverting attention away from the pain. However, the exact neural mechanisms of VR are as yet unknown and can be only speculated upon $[18,19]$.

Keefe et al. state that while there is growing evidence supporting VR's effectiveness in managing acute procedural pain, little is known about the use of VR for chronic pain [20]. Chronic pain is known to be substantially different from acute pain [21]. Multiple variables are involved with the sensation of chronic pain, including various psychological factors and central nervous system processes. It is possible that virtual reality, while having been shown to be effective for acute pain, is ineffective for chronic pain due to these factors.

\subsection{Use with Chronic Pain}

A review of the current literature finds that there have been only two studies to date assessing the impact of VR on chronic pain $[22,23]$. In the first study, participants were asked about their pain before a VR session and while they were in the VR session. The session was 15 minutes long and used pleasant and relaxing scenes. The study found a significant reduction in reported pain which was corroborated by physiological measures. The study did not investigate the impact of VR on chronic pain after the VR session was over. The second study, done by our research group, assessed the impact of a five-minute VR session on chronic pain levels during the session and immediately afterwards. That study showed a significant reduction in pain during the session and immediately afterwards

The two studies on chronic pain, taken with the many studies on acute pain, suggest that VR sessions can have a significant impact in the moment on chronic pain levels. The next question then is how VR could be used to help those who suffer from chronic pain to extend the period of pain relief. As chronic pain is an ongoing and enduring experience, the hope is that VR could impact chronic pain in an enduring manner. The purpose of the present study was to learn more about the possible impact of VR sessions on chronic pain patients beyond immediate analgesia. The intervention was designed to be similar to a previous study on the effectiveness of mindfulness-based stress reduction (MBSR) for acute pain [25]. This study found that three 20 minute sessions of MBSR training had a positive impact on the experience of acute pain. It was hoped that an intervention using a similar amount of time would be successful as well, and this explains why we 
choose this amount of time for our intervention.

This current pilot study was designed to help guide future research in how VR sessions might have positive impacts on the chronic pain experience. In this study, we looked at such variables as strength and duration of analgesia, side effects of the VR session, depression, anxiety, catastrophizing and perceived control in coping with pain. The study took a small sample of subjects with similar pain diagnoses and used multiple psychological assessment measures to look for possible impacts and to help guide future more rigorous studies.

\section{METHODS}

The study was approved by IntegReview, an independent IRB located in Austin, Texas. Participants were recruited from an outpatient pain practice in Knoxville, Tennessee (Pain Consultants of East Tennessee). Recruitment flyers were placed in the practice lobby and in the physical therapy area. Participation in the study was voluntary and had no bearing on the patient's pain treatment.

\subsection{Inclusion}

To qualify for the study, participants had to be at least 18 years old, must not be visually or hearing impaired, had to be an active patient at the pain practice, had to have had an initial psychological assessment, and had to have been assessed at the initial psychological assessment as having sufficient cognitive faculties to give informed consent. They also needed to have a primary diagnosis involving a neuropathic pain condition. Diagnoses which were acceptable for the study included a primary diagnosis of
Chronic Regional Pain Syndrome (CRPS), peripheral neuropathy, small fiber neuropathy, phantom limb syndrome or trigeminal neuralgia. While involving different parts of the body, these diagnoses were selected as they all represent a chronic neuropathic pain condition. This was done in order to have a fairly uniform patient sample. Participants who met the eligibility criteria and agreed to be in the study were then given the informed consent form to review and sign.

\subsection{Design}

The study design is outlined in Figure 1. The study used three 20-minute sessions of VR as the intervention. These were offered on three consecutive weeks. Data were gathered about the subject's pain after each of these sessions and one week after the third VR session. These data gathering points were termed "Time 1," "Time 2," "Time 3," and "Time 4." A packet of psychology assessments was administered before the first VR session (Time 1) and one week after the third VR session (Time 4). A cash incentive of fifty dollars was given to all participants after each of the five study sessions were completed.

\subsection{VR Intervention}

When the VR session occurred, the equipment and the general visual experience were explained to the participant and all questions were answered. Before the VR session started he or she was asked what his or her pain level was on the $0-10$ numerical rating scale. The participant then donned the virtual reality headset and headphones. Some participants used the Oculus Rift system to use the application (described below) while others used the Vive system to use the application. This selection was not done randomly. Early study participants used the 
Oculus system. A Vive system was obtained in the midst of the study. From that point on all participants used the Vive system. When using the Oculus system participants were given a mouse and a clipboard to interact with the application. When using the Vive participants were given the two hand controllers. The participant then engaged in the virtual reality experience (described below) for twenty minutes. After the 20minute experience the VR equipment was removed and the participant was asked what his or her pain was on the 0-10 numerical rating scale at that moment. The participant was then asked what his or her pain level was during the virtual reality experience, again using the numerical rating scale.

Participants were then asked two questions about engagement in the virtual reality experience: "On a scale of $0-10$, to what extent did you feel like you went inside the virtual world? Ten is 'I went completely inside the virtual world' and 0 is ' $\mathrm{I}$ did not feel like I went inside it at all' and also: "On a scale of $0-10$, how real did the objects seem to you? Ten is 'indistinguishable from the real world' and 0 is 'completely fake." Participants were also asked three questions about any side effects: "On a scale of 0-10 how much dizziness did you feel while you were in the virtual world? Ten is 'feel faint' and zero is "no dizziness at all", and "On a scale of 0-10 how bad a headache did you feel while you were in the virtual world? Ten is 'worst headaches possible' and zero is 'no headache at all' and 'On a scale of 0-10 how much nausea did you feel while you were in the virtual world? Ten is 'vomited' and zero is "no nausea at all."

The VR application used is these studies is called COOL! (FirstHand Technology, Inc., 2014). COOL! is an interactive journey through a fully immersive $360^{\circ}$ VR fantasy landscape (see Jones et al, 2016 for more complete description). Participants are taken along a route through a virtual landscape (see Figure 2 for screen shots and a link to a video of the experience). Once started the participant "travels" through the landscape at a constant speed until the application automatically stops at the time limit (twenty minutes). Participants can interact with various aspects of the landscape as they "travel" using the buttons of a mouse (Oculus) or hand controllers (Vive). Participants can shoot bubbles and throw fish at environment objectives with some environmental reactivity (sounds, movement and color changes). There is no violence involved. There is no score to be kept. Participants were assured that they could stop any time for any reason but none did and all participants had the full 20-minute experience.

\subsection{Assessment Measures}

Pain intensity was measured with a $0-10$ numerical rating scale of pain. A $0-10$ numerical rating of pain has been shown to be a valid and reliable method of rating pain intensity and shows better compliance, responsiveness and ease of use compared to verbal rating scales or visual analog scales [25]. The anchors for the numerical rating scale were "Ten is the worst pain anyone could ever have and zero is no pain at all." When the study design called for an assessment to be done, before the VR sessions participants were asked several questions about their pain. This included pain average for the last month and pain maximum and minimum in the past month, pain average, maximum and minimum for the last week, and current pain level. They were also asked about their pain at the follow-up session (Time 4) when no VR was administered. 
The study hypothesis was that receiving analgesia from VR might also lead to a decrease in depression, anxiety and/or catastrophizing and possibly an increase in a feeling of self-efficacy in dealing with chronic pain. Four psychological measures were administered at two intervals during the study (see Figure 1). These included the Zung Depression Scale [26], the Zung Anxiety Scale [27], the Pain Catastrophizing Scale [28], and the Chronic Pain Self-Efficacy Scale [29]. The four inventories are all validated measures for their domains. The Zung Depression Scales and the Zung Anxiety Scale, as the names indicate, are validated measures of depression and anxiety, respectively. The Pain Catastrophizing Scale measures the construct of "catastrophizing." Catastrophizing has been shown to be a significant and primary predictor of outcomes in pain treatment [30]. The Chronic Pain Self-Efficacy Scale is a measure of self-efficacy - the belief that one has control and power to deal with chronic pain. This belief in one's ability to cope is thought to be an important one in predicting outcomes of various chronic pain conditions [31].

Finally, at the second, third and fourth sessions the participants were asked if he or she thought the previous VR experience had helped with their pain, rated as "Made it worse," "No help," "Helped a little," "Helped moderately," and "Helped a great deal." They were asked "If VR helped your pain, about how long would you say it helped your pain afterwards?" and were asked to estimate this in hours or days (or not at all).

\section{RESULTS}

A total of ten subjects comprised the study sample. Two additional subjects enrolled but did not complete all three VR sessions and were replaced with subjects who did complete all three sessions. Data from the two dropouts were not included in these analyses. The ten subjects who comprised this study's subject population were middle aged $(M=56.30, S D=9.62)$, half $(50 \%)$ were female, and all were married. Fifty percent had a diagnosis of CRPS, while $30 \%$ had a diagnosis of idiopathic neuropathy and one subject each was diagnosed with small fiber neuropathy and diabetic peripheral neuropathy.

\subsection{Impact on Pain}

Pain ratings were obtained from each subject before and after each VR session. Subjects were also asked after the session what their pain was during the session. Figure 3 displays a graph of the average pain ratings before, during and immediately after each of the three VR sessions. Pain before each VR session averaged 5.1, during the session it averaged 1.8, and after the session the average rating was 2.8. Paired samples t-test comparisons (Table 1) revealed significant pain reductions during and immediately after each session. Pain scores during the session were $65 \%$ less than before the session, while pain scores after the session were $45 \%$ less than before the session. There is no indication that habituation occurred, as session 3 analgesia appears as effective as session 1 . Earlier VR sessions used the Oculus Rift (21 sessions) while later VR sessions used the Vive (9 sessions). There was no statistical difference between the two systems on their impact on changes in pain.

The average rating to the question "On a scale of $0-10$, to what extent did you feel like you went inside the virtual world?" was 8.3. This indicates that subjects were, on average, successfully immersed in the VR experience. 
The average rating for the question "On a scale of $0-10$, how real did the objects seem to you?" was 6.5 also indicating that subjects achieved immersion in this application.

Subjects were asked at follow-up sessions after VR experiences if they thought the VR session had decreased their pain, and if so, how long had it helped. The average response was "helped moderately" (3.9 on a five point scale). Subjects reported $97 \%$ of the time that they thought VR had helped their pain with only one subject in one session reporting that it did not help pain. The average estimate of how long the analgesia had occurred after the session was 30 hours. Ten percent of the time (three of thirty responses) subjects reported that there was no aftereffect of VR on their pain while $90 \%$ of the time there was some aftereffect, ranging from a minimum 30 minutes to a maximum of 72 hours.

All subjects were asked to rate their average pain for the last month, the last week and the current time at sessions 1, 2, 3 and 4 before VR was done. Data on pain ratings across time are displayed in Figure 5, with one line for average pain in the last month, one line for average pain in the past week, and one line for current pain. These data show a statistically significant decrease in average monthly pain rating (Time 1 to Time 4), $[\mathrm{t}(7)=4.25, \mathrm{p}<.01)]$. Average weekly and current pain changes across time (Time 1 to Time 4) were not statistically significant.

\subsection{Side Effects}

As to side effects, participants were asked three questions about any side effects regarding dizziness, headache and nausea. The average ratings were for these three questions were $.3, .1$, and .4 respectively. This indicates that side effects were minimal to non-existent for almost subjects. One subject of the ten reported side effects more frequently than any other subject, and accounted for $86 \%$ of all the side effects reported. Only one other subject reported any side effect of any kind during the sessions. No subject requested that the VR experience be stopped or modified in any way though they were told they could ask for this before the session was started.

\subsection{Impact on Psychological Variables}

All subjects were administered psychological assessment tools at Time 1 and at Time 4. These data are displayed graphically in Figure 5 with group average scores for depression anxiety, catastrophizing and feelings of control over the pain. There were no statistically significant differences on any of the four measures from Time 1 to Time 4 .

\section{SUMMARY AND DISCUSSION}

This pilot study used three weekly 20 -minute VR sessions using the application Cool! as an intervention for chronic neuropathic pain in ten subjects. Analysis of the data here finds that the subjects reported significant analgesia during and immediately after the VR session. The average decrease in pain during the session was $65 \%$ and the average decrease in pain immediately after the session was $45 \%$. In a previous study by our group [23] using five minute VR sessions with the same application, the average analgesia was $60 \%$ during the session and $33 \%$ immediately afterwards. Our data here then suggest that longer VR sessions yields a slightly larger overall degree of analgesia than shorter VR sessions. This study also found that there may be some "tail" or aftereffect to the VR session's analgesia, with subjects here reporting an average decrease in pain for 30 hours. Side effects of the VR experience here 
were negligible for most subjects and immersion was good.

Average pain scores for the last month, the last week, and at the present moment were gathered across time. Subjects' rating of average pain for the month decreased significantly from the beginning of the study to one week after the intervention period. However, ratings of current pain and weekly pain did not change significantly. Based on the study design we would have expected weekly and current pain ratings to change if the treatment was having an ongoing impact. As the study was conducted over a four week period for each subject, a change in ratings of average pain over the last month does not seem attributable to the intervention. As current pain ratings did not change in a statistically significant manner but did trend in the downward direction, more study on this indicated.

Overall, at least for the VR application Cool!, while it offers very good analgesia during the experience and for a somewhat brief time afterwards, these data do not show a major, if any, impact on chronic pain over time resulting from three repeated weekly 20minute sessions. Measurement of depression, anxiety, catastrophizing and sense of control over pain did not appear to change after the three-session intervention. The application Cool! is modeled after Snow World, the much-studied application found to have significant analgesia for burn pain and procedural pain. This current study suggests that programs of this type - ones that provide significant analgesia through distraction and multi-sensory override of pain - may have limits when it comes to use with chronic pain. Cool! has now been shown in two studies to date to provide very good analgesia for chronic pain at the time of VR administration, and that there is some aftereffect to this analgesia of about 30 hours. However, providing repeated analgesia for chronic pain may not have a significant long-term impact on chronic pain levels, and most pain ratings were statistically unchanged after this threeweek intervention was complete. The repeated analgesia also did not impact important psychological variables such as depression, anxiety, catastrophizing or sense of control over pain. In sum, current VR programs for pain such as Cool! do what they do and they do it very well - they offer very good immediate analgesia for pain, both for acute and for chronic pain. However, the provision of repeated analgesia does not appear to have secondary effects that result in any fundamental changes in the neurosensory, emotional or cognitive aspects of the chronic pain experience.

\subsection{Implications}

It may be that new applications need to be developed to best treat chronic pain. These applications would need to offer more than "mere" analgesia and instead, or in addition, provide some sort of skill training to better address the associated aspects of chronic pain. Applications that teach relaxation skills, combat catastrophizing or teach mindfulnessbased stress reduction (MBSR) are examples of what VR seems to need to offer as an intervention for chronic pain conditions. Alternatively, longer or more frequent VR sessions would be another way for VR to be used for chronic pain. It could be that daily in-home sessions of VR could provide effective analgesia and would not necessarily need to teach skills. The VR sessions done here were in-office treatments and the equipment (Oculus Rift and Vive) is financially out of reach for most pain patients. Applications that could provide analgesia using a Gear, Goggle Daydream or Google cardboard could perhaps offer 
repeated and daily analgesia for chronic pain patients which could have ongoing benefit.

\subsection{Limitations}

The current study used a very small sample. Funding limitations precluded having a larger sample size. Future research should use larger study samples to confirm or deny the findings of this study. It could well be that studies with larger sample sizes may find impacts that the current study missed. A more frequent or intensive VR intervention and a longer follow-up period would be useful in looking at VR impact on chronic pain. As this was an exploratory study two different VR systems were used during the course of the study. This is not an optimal study design but this was done in an effort to gather as much data about VR and its impact as possible. While no statistical differences were found here in sessions done with the Oculus Rift versus the Vive, future studies should be more controlled and use one system throughout a study.

Studies of VR on other chronic pain conditions is recommended. Neuropathic pain is one subset of a host of chronic pain conditions and use of VR with other pain conditions should be explored. Finally, the current study also only used one VR application for pain (Cool!). While Cool! and its forerunner, Snow World, have been widely studied for their impact on pain, investigation of other VR applications is needed and could offer useful information about what aspects of the VR experience were post effective in treating chronic pain. Overall, there are multiple limits to this pilot study and the results here should be viewed as preliminary rather than definitive.
The world of pain treatment in general is undergoing a revolution as it transitions from a past reliance on opioid medications to other non-opioid treatments. VR could have an important role in play in offering ongoing analgesia for patients with chronic pain. This study hopefully helps guide future in the use of VR for chronic pain, as we work to find effective and affordable non-opioid treatments for these widespread conditions.

\section{REFERENCES}

[1]. Federation of State Medical Boards. (2013). Model policy on the use of opioid analgesics in the treatment of chronic pain. Washington, DC: The Federation.

[2]. Noble, M., Treadwell, J., Tregear, S., Coates, V., Wiffin, P., Akafomo, C., \& Schoelles, K. (2010). Long-term opioid management for chronic noncancer pain. Cochrane Database of Systematic Reviews, Issue 1. New York, NY: The Cochrane Collaborative, John Wiley \& Sons, Ltd.

[3]. Rosenblum, A., Marsch, L., Joseph, H., \& Portenoy, R. (2008). Opioids and the treatment of chronic pain: controversies, current status, and future directions. Experimental and Clinical Psychopharmacology, 16 (5): 405-416.

[4]. Office of National Drug Control Policy. (2011). Epidemic: responding to America's prescription drug abuse crisis. Washington, DC: Executive Office of the President, The White House.

[5]. Gershon, J., Zimand, E., Pickering, M., Rothbaum, B., \& Hodges, L. (2004). A pilot and feasibility study of virtual reality as a distraction for children with cancer. Journal of the American Academy of Child and Adolescent Psychiatry, 43(10), 1243-9.

doi:10.1097/01.chi.0000135621.231 45.05 . 
[6]. Hoffman, H., Patterson, D., \& Carrougher, G. (2000). Use of virtual reality for adjunctive treatment of adult burn pain during physical therapy: a controlled study. Clinical Journal of Pain, 16(3), 244-250.

[7]. Hoffman, H., Patterson, D., Carrougher, G., \& Sharar, S. (2001). Effectiveness of virtual reality-based pain control with multiple treatments. Clinical Journal of Pain, 17(3), 229-35. Retrieved from http://www.ncbi.nlm.nih.gov/pubme d/11587113

[8]. Hoffman, H., Patterson, D., Seibel, E., Soltani, M., Jewett-Leahy, L., \& Sharar, S. (2008). Virtual reality pain control during burn wound debridement in the hydrotank. Clinical Journal of Pain, 24(4), 299304.

doi:10.1097/AJP.0b013e318164d2c c

[9]. Chan, E., Chung, J., Wong, T., Lien, A., \& Yang, J. (2007). Application of a virtual reality prototype for pain relief of pediatric burn in Taiwan. Journal of Clinical Nursing, 16(4), 786-93. doi:10.1111/j.13652702.2006.01719.x

[10]. Maani, C., Hoffman, H., DeSocio, P., Morrow, M., Gaylin, C., Magula, J. \& Gaylord, K. (2008). Pain control during wound care for combatrelated burn injuries using custom articulated arm mounted virtual reality goggles. Journal of Cyber Therapy and Rehabilitation, 1(2), 193. Retrieved from http://citeseerx.ist.psu.edu/viewdoc/ download?doi=10.1.1.192.9242\&am p;rep=rep1\&amp;type $=$ pdf

[11]. Maani, C., Hoffman, H., Morrow, M., Maiers, A., Gaylord, K., McGhee, L., \& DeSocio, P. (2011). Virtual reality pain control during burn wound debridement of combatrelated burn injuries using robot-like arm mounted VR goggles. Journal of Trauma, 71(1 Suppl), S125-30. doi:10.1097/TA.0b013e31822192e2
[12]. Mosso, J., Obrador, G., Wiederhold, B., Wiederhold, M., Lara, V., \& Santander, A. (2012). Cybertherapy in medicine-experience at the Universidad Panamericana. In $\mathrm{C}$. Eichenberg (Ed). Virtual reality in psychological, medical and pedagogical applications. Published September 12, 2012. ISBN 978-95351-0732-3.

[13]. Patterson, D., Hoffman, H., Palacios, A., \& Jensen, M. (2006). Analgesic effects of posthypnotic suggestions and virtual reality distraction on thermal pain. Journal of Abnormal Psychology, 115, 834-841. doi:10.1037/0021-843X.115.4.834

[14]. Sharar, S., Carrougher, G., Nakamura, D., Hoffman, H., Blough, D., \& Patterson, D. (2007). Factors influencing the efficacy of virtual reality distraction analgesia during postburn physical therapy: preliminary results from 3 ongoing studies. Archives of Physical Medicine and Rehabilitation, 88(12 Suppl 2), S43-9. doi:10.1016/j.apmr.2007.09.004

[15]. VanTwillert, B., Bremer, M., \& Faber, A. (2007). Computergenerated virtual reality to control pain and anxiety in pediatric and adult burn patients during wound dressing changes. Journal of Burn Care Research, 28(5), 694-702. doi:10.1097/BCR.0B013E318148C 96F

[16]. Melzack, R., \& Wall, P. (1965). Pain assessment, a new theory. Science, 150, 971-5.

[17]. Gold, J., Belmont, K., \& Thomas, D. (2007). The neurobiology of virtual reality pain attenuation. Cyberpsychology and Behavior, 10(4), 536-44. doi:10.1089/cpb.2007.9993

[18]. Gold, J., Belmont, K., Thomas, D. (2007). The neurobiology of virtual reality pain attenuation. Cyberpsychology and Behavior, 10(4):536-544. 
[19]. Li, A., Montaño, Z., Chen, V., \& Gold, J. (2011). Virtual reality and pain management: current trends and future directions. Pain Management, 1(2), 147-157.

[20]. Keefe, F., Huling, D., Coggins, M., Keefe, D., Zachary Rosenthal, M., Herr, N., \& Hoffman, H. (2012). Virtual reality for persistent pain: a new direction for behavioral pain management. Pain. 153(11):21632166.

doi:10.1016/j.pain.2012.05.030.

[21]. Grichnik, K., \& Ferrante, F. (1991). The difference between acute and chronic pain. Mt. Sinai Journal of Medicine, May;58(3):217-20.

[22]. Wiederhold, B., Gao, K., Sulea, C., \& Wiederhold, M. (2014). Virtual reality as a distraction technique in chronic pain patients. Cyberpsychology,

Behavior, and Social Networking 17(6):346-352.

doi:10.1089/cyber.2014.0207.

[23]. Jones, T., Moore, T., \& Choo J. (2016). The impact of virtual reality on chronic pain. PLOS ONE. December 20, 2016. https://dx.doi.org/10.1371/journal. pone.0167523.

[24]. Hjermstad, M., Fayers, P., Haugen, D., Caraceni, A., Hanks, G., Loge, J., Fainsinger, R., Aass, N., Kaasa, S. (2011). Studies comparing numerical rating scales, verbal rating scales, and visual analogue scales for assessment of pain intensity in adults: a systematic review. Journal of Pain and Symptom Management, 41(6):1073-93.

[25]. Zeidan, F., Gordon, N., Merchant, J., \& Goolkasian, P. (2010). The effects of brief mindfulness meditation training on experimentally induced pain. Journal of Pain, 11(3): 199209.

[26]. Zung, W. (1965). A self-rating depression scale. Archives of General Psychiatry, 12: 63-70.
[27]. Zung, W. (1971). A rating instrument for anxiety. Psychosomatics, XII: 371-379.

[28]. Sullivan, M., Bishop, S., Pivik, J. (1995). The Pain Catastrophizing Scale: development and validation. Psychological Assessment, 7: 524-532.

[29]. Anderson, K., Dowds, B., Pelletz, R., Edwards, W., \& $\underline{\text { Peeters- }}$ Asdourian C. (1995). Development and initial validation of a scale to measure self-efficacy beliefs in patients with chronic pain. Pain 63(1): 7783.

[30]. Craner, J., Sperry, J., Evans, M. (2016). The relationship between pain catastrophizing and outcomes of a 3-week comprehensive pain rehabilitation program. Pain Medicine, 17 (11): 2026-2035.

[31]. Somers, T., Wren, A., \& Shelby, R. (2012). The context of pain in arthritis: self-efficacy for managing pain and other symptoms. Current Pain and Headache Reports, 16(6), 502508. 
Psychological Assessment

\begin{tabular}{|c|c|c|c|}
\hline Pain Assessment & Pain Assessment & Pain Assessment & Pain Assessment \\
\hline $1^{\text {st }} V R$ & $2^{\text {nd }} V R$ & $3^{\text {rd }} V R$ & Follow-up \\
\hline Start & 1 week later & 1 week later & 1 week later \\
\hline Time 1 & Time 2 & Time 3 & Time 4 \\
\hline
\end{tabular}

Figure 1. Study design $(\mathrm{N}=10)$ 

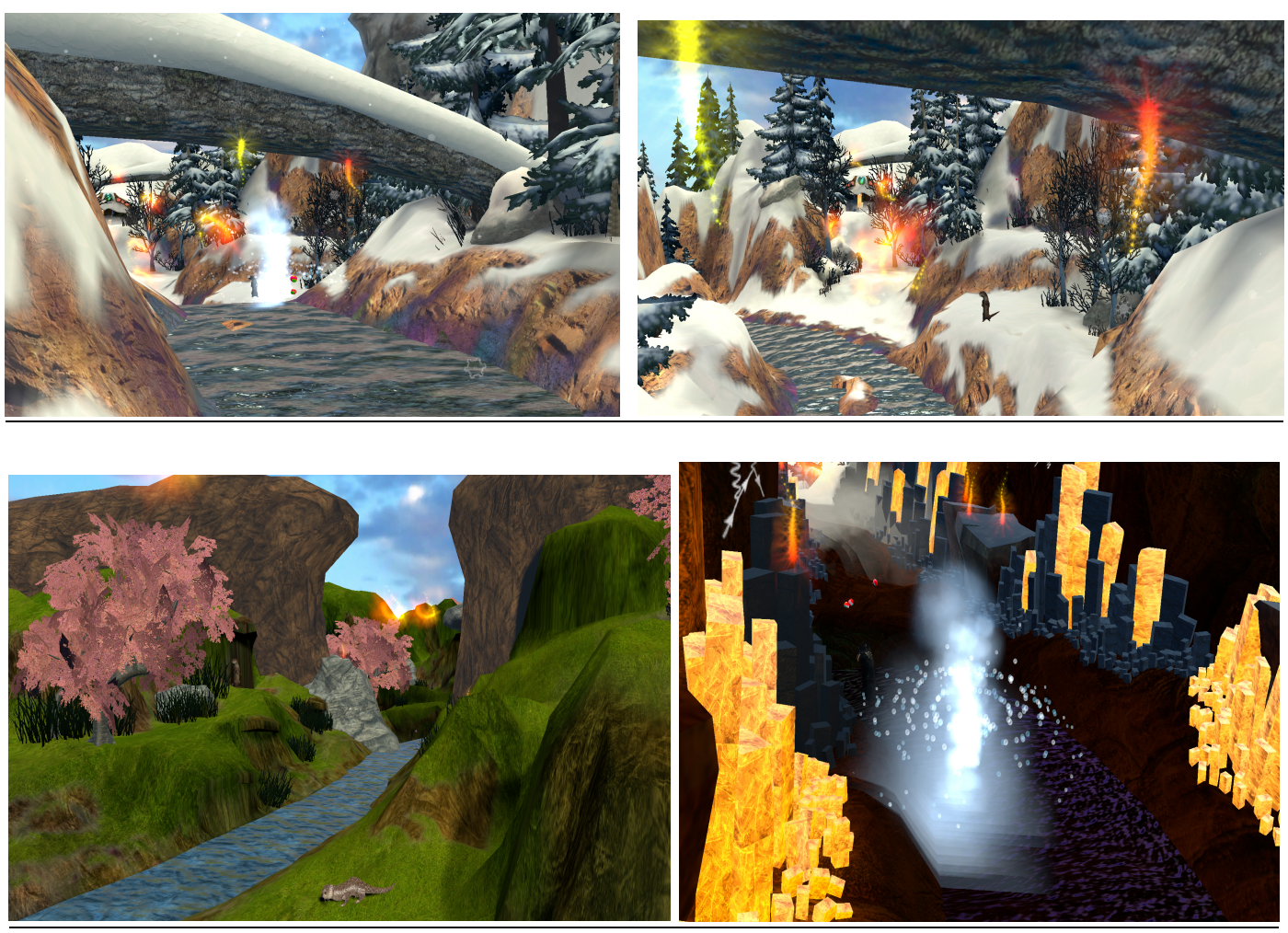

Figure 2. Screen shots and video from Cool!

Link to a video of Cool!: https://www.youtube.com/watch?v=9vzwr_loFx8 


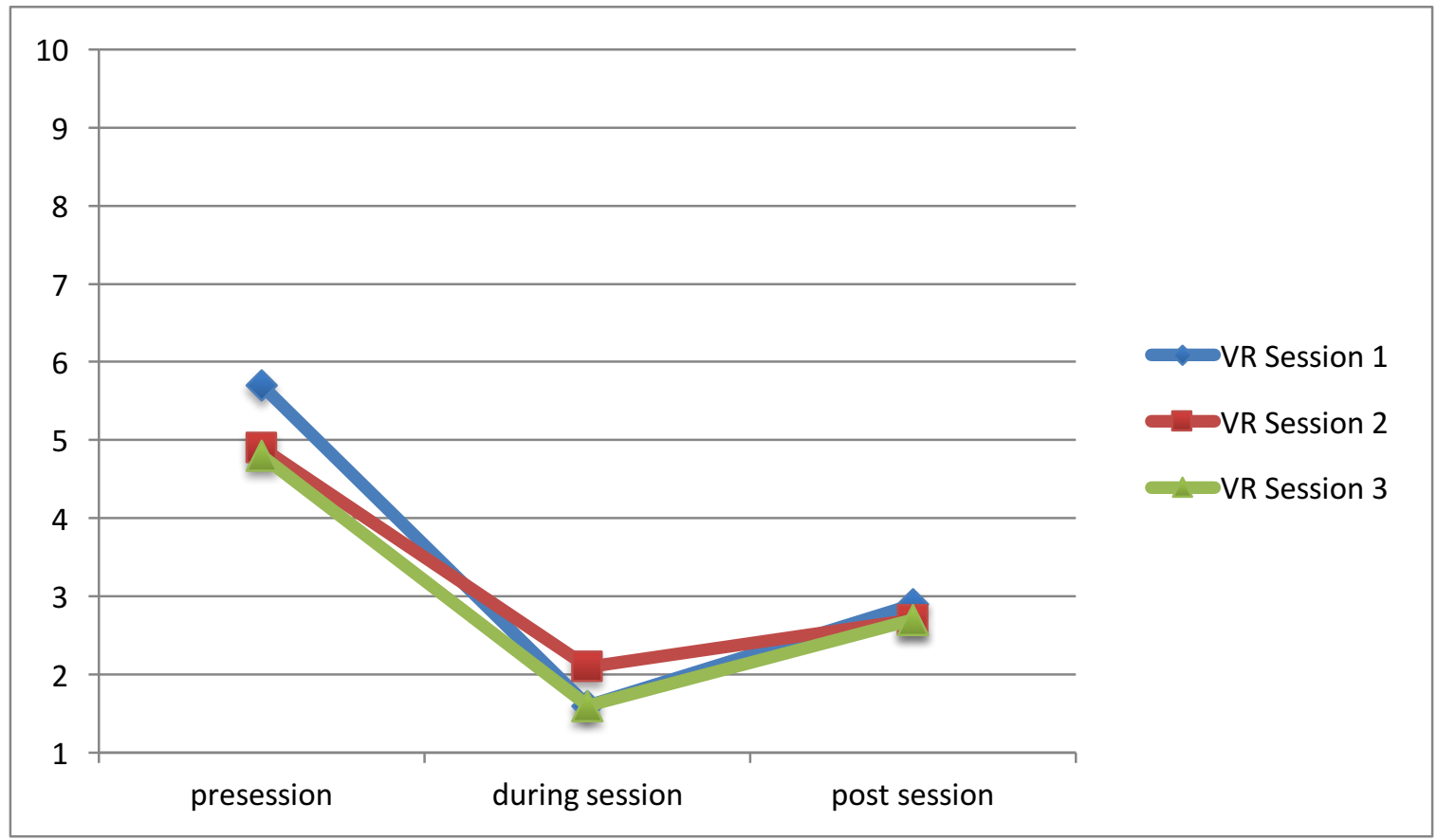

Figure 3. Average pain ratings before, during and immediately after the vr session 


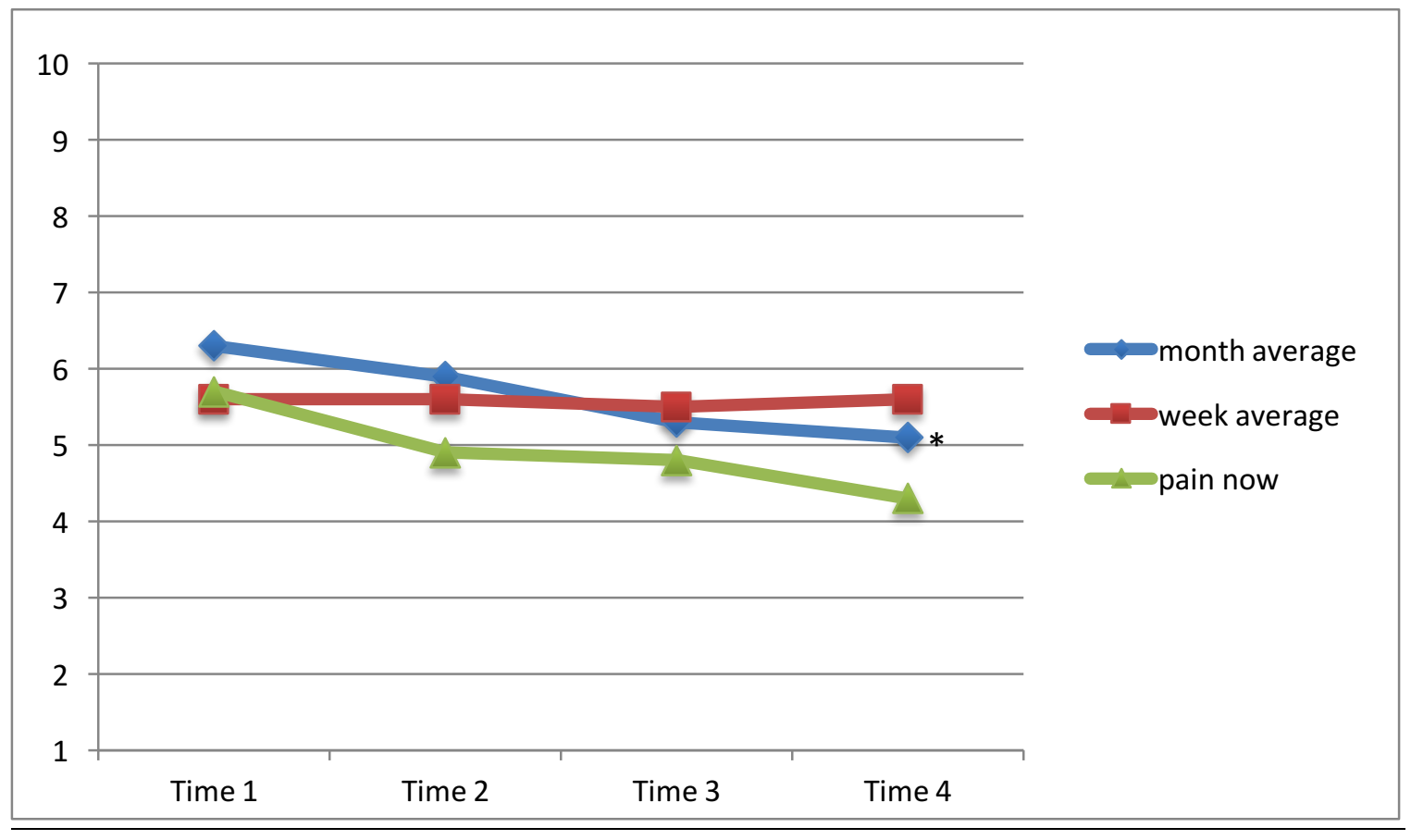

* Monthly pain rating showed a statistically significant change Time 1 to Time 4 . No other changes in pain were statistically significant.

Figure 4. Average pain ratings across time 


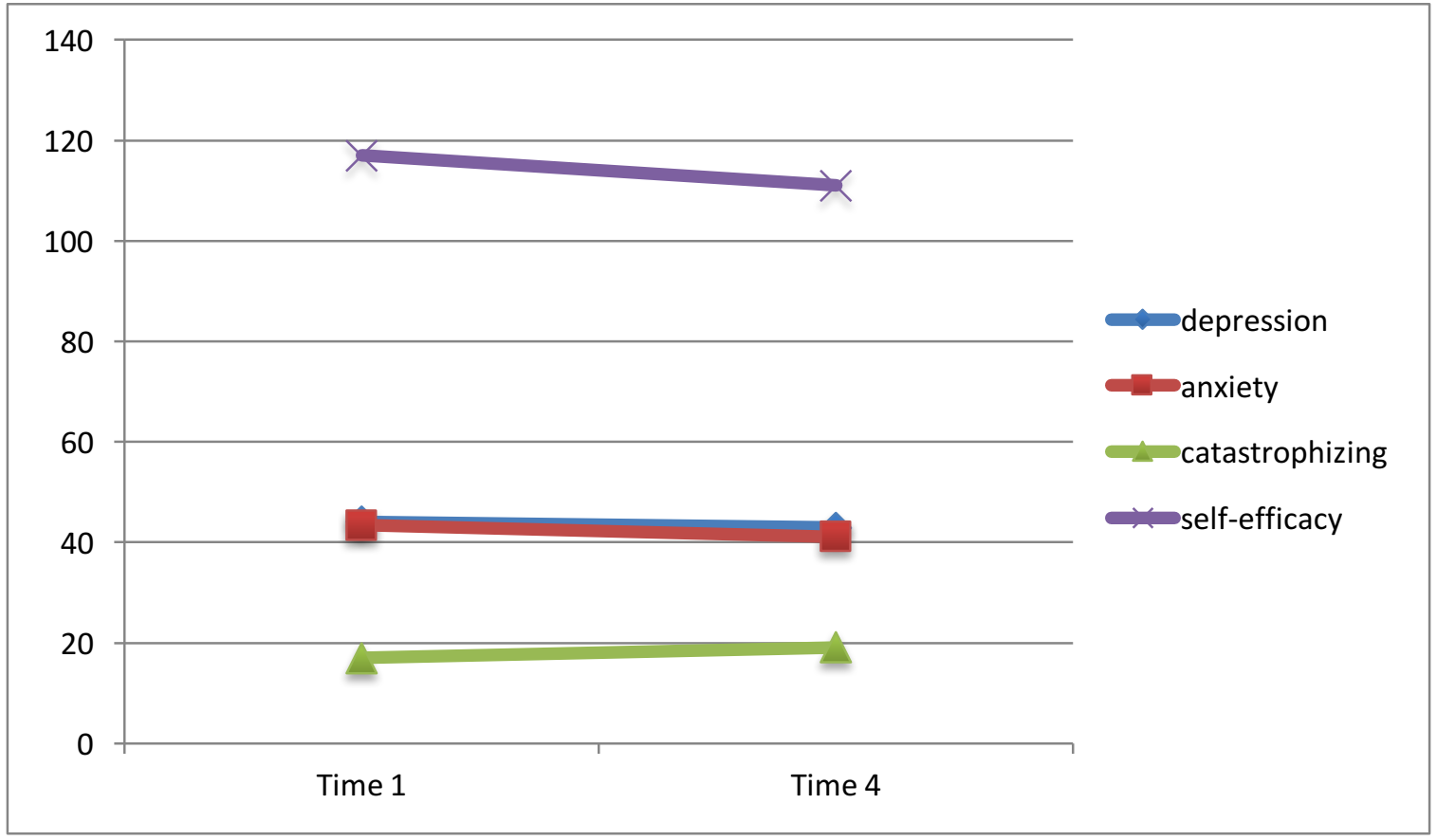

Figure 5. Psychological assessment scores across time 
Table 1

T-Test Comparisons of Pain Scores at Each Session

\begin{tabular}{|c|c|c|c|c|c|c|c|c|}
\hline & \multicolumn{2}{|c|}{ Pre-treatment } & \multicolumn{2}{|c|}{$\begin{array}{c}\text { During } \\
\text { Treatment }\end{array}$} & \multirow{2}{*}{$\frac{\frac{\text { Pre-During }}{\text { Comparison }}}{t(9)}$} & \multicolumn{2}{|c|}{$\stackrel{\text { Post- }}{\text { treatment }}$} & \multirow{2}{*}{$\frac{\frac{\text { Pre-Post }}{\text { Compariso }}}{\frac{\mathrm{n}}{t(9)}}$} \\
\hline & $M$ & $S D$ & $M$ & $S D$ & & $M$ & $S D$ & \\
\hline Treatment 1 & 5.70 & 2.87 & 1.60 & 1.71 & $5.16^{* *}$ & 2.90 & 1.85 & $4.22 * *$ \\
\hline Treatment 2 & 4.89 & 2.13 & 2.10 & 2.02 & $5.47 * *$ & 2.70 & 1.95 & $4.71 * *$ \\
\hline Treatment 3 & 4.80 & 2.04 & 1.60 & 1.71 & $4.60 * *$ & 2.70 & 1.42 & $3.99 * *$ \\
\hline
\end{tabular}

Note: $* * p<.01$

Pain scores before VR session, during the session and after the session. $t$ test compares changes in pain between pain before and pain during the session, also pain before and pain after the session, for each treatment session. 